\title{
Community pharmacy-based asthma services: current perspectives and future directions
}

This article was published in the following Dove Press journal:

Integrated Pharmacy Research and Practice

II August 2014

Number of times this article has been viewed

\author{
Suzanne G Bollmeier \\ Theresa R Prosser \\ St Louis College of Pharmacy, \\ St Louis, MO, USA
}

Correspondence: Suzanne G Bollmeier St Louis College of Pharmacy, 4588 Parkview Place, St Louis, MO 63।I0, USA

$\mathrm{Tel}+\mathrm{I} 3144468525$

Fax + I 3144468500

Email sbollmeier@stlcop.edu

\begin{abstract}
Achieving and maintaining asthma control and improving patients' quality of life are cornerstones of asthma management. This review summarizes the current literature related to services provided by community pharmacists to patients with asthma. Comprehensive asthma programs provided by community pharmacists have improved patients' knowledge of the disease, device technique skills, patient adherence, and quality of life. One study shows such comprehensive programs are cost effective in patients with severe or uncontrolled asthma, which cannot be extrapolated to all programs. Targeted interventions by pharmacists could be provided to a larger population of patients. Pharmacists have identified that lack of time, resources, and training are barriers to implementing asthma programs. In addition, optimal models are needed to integrate interventions into the dispensing workflow. Optimal training programs should include skills in problem solving, device technique, and counseling. A movement towards "institutionalizing" routine asthma interventions or patient encounters is necessary if consistent services are to be given to all patients, and appropriate compensation is provided for pharmacist services.
\end{abstract}

Keywords: community, pharmacy, pharmacist, asthma, education

\section{Introduction}

It is estimated that 18.7 million adults $(8.2 \%)$ and seven million children $(9.4 \%)$ in the United States have asthma, and that 29.1 million adults $(12.7 \%)$ and 10.1 million children (13.6\%) will be diagnosed with asthma during their lifetimes. ${ }^{1}$ The prevalence of asthma has risen by $14.8 \%$ in less than 10 years (2001-2010). ${ }^{1}$ The National Asthma Education and Prevention Program: Expert Panel Report 3 guidelines ${ }^{2}$ call for asthma self-management education to be integrated into all aspects of care. Healthy People $2020^{3}$ highlights the need to increase the proportion of patients receiving care according to asthma guidelines, written asthma management plans, and formal patient education in order to improve asthma outcomes and reduce asthma-related urgent care visits and deaths.

Pharmacist-driven intervention programs for respiratory disorders have been documented to decrease symptoms and improve asthma severity. ${ }^{4}$ However, many of these programs are clinic- or institutionally based. Fewer programs are situated in a community pharmacy. The challenge for community pharmacists is to fully translate the potential of their therapeutic knowledge and skills into consistently provided programs that improve asthma control. The general workflow is different between community and clinic pharmacy practice. Community pharmacists have access to dispensing data, but they generally have more limited access to patient medical information. Effective strategies based on the available patient data, necessary resources, and 
methods to overcome barriers to implementing community pharmacy-based asthma programs need to be identified. It would also be important to assess the acceptance of such programs by patients and providers.

The aim of this review is to summarize the data regarding community pharmacist-provided asthma care. Based on these findings, our goal is to identify insights and make recommendations to promote a broader, more consistent involvement of community pharmacists in asthma management, so as to increase their impact on asthma outcomes.

\section{Data sources}

An initial PubMed (National Center for Biotechnology Information, US National Library of Medicine, Bethesda, MD, USA), Embase (Elsevier BV, Philadelphia, PA, USA), and International Pharmaceutical Abstracts (Thomson Scientific, Inc., Philadelphia, PA, USA) database search was conducted in January 2014 for clinical trials using the term "asthma" and each of the following: "community pharmacy"; "pharmacist"; "interventions"; "pharmaceutical care"; "pharmaceutical services"; "medication management"; and "medication therapy services". There were no time limits. Additional publications were identified by reviewing the references of the identified trials.

\section{Study selection/data extraction}

All types of trials (for example, randomized controlled trials [RCTs], surveys, pilot, and feasibility trials) were included, as long as the study clearly noted that community pharmacists or community pharmacy practice was the focus of the program. All trials published in English were included regardless of the country of origin. Trials also including subjects with chronic obstructive pulmonary disease (COPD) were included if the intervention and measures used were applicable to both asthma and COPD. A recent publication ${ }^{5}$ summarized the results from trials published between 1997-2008 involving community pharmacists. Thus, for trials related to serial community pharmacist-patient encounters for asthma care, this review focuses on those subsequent to that publication (ie, April 2008-December 2013). All other trials were included regardless of publication date. Studies were excluded if not in English or if they did not clearly identify the intervention as involving community pharmacists.

\section{Results}

Thirty-three studies were identified (see Figure 1), and categorized as follows:

- Community pharmacist-patient encounters for asthma (post-2008): number (n)=7.
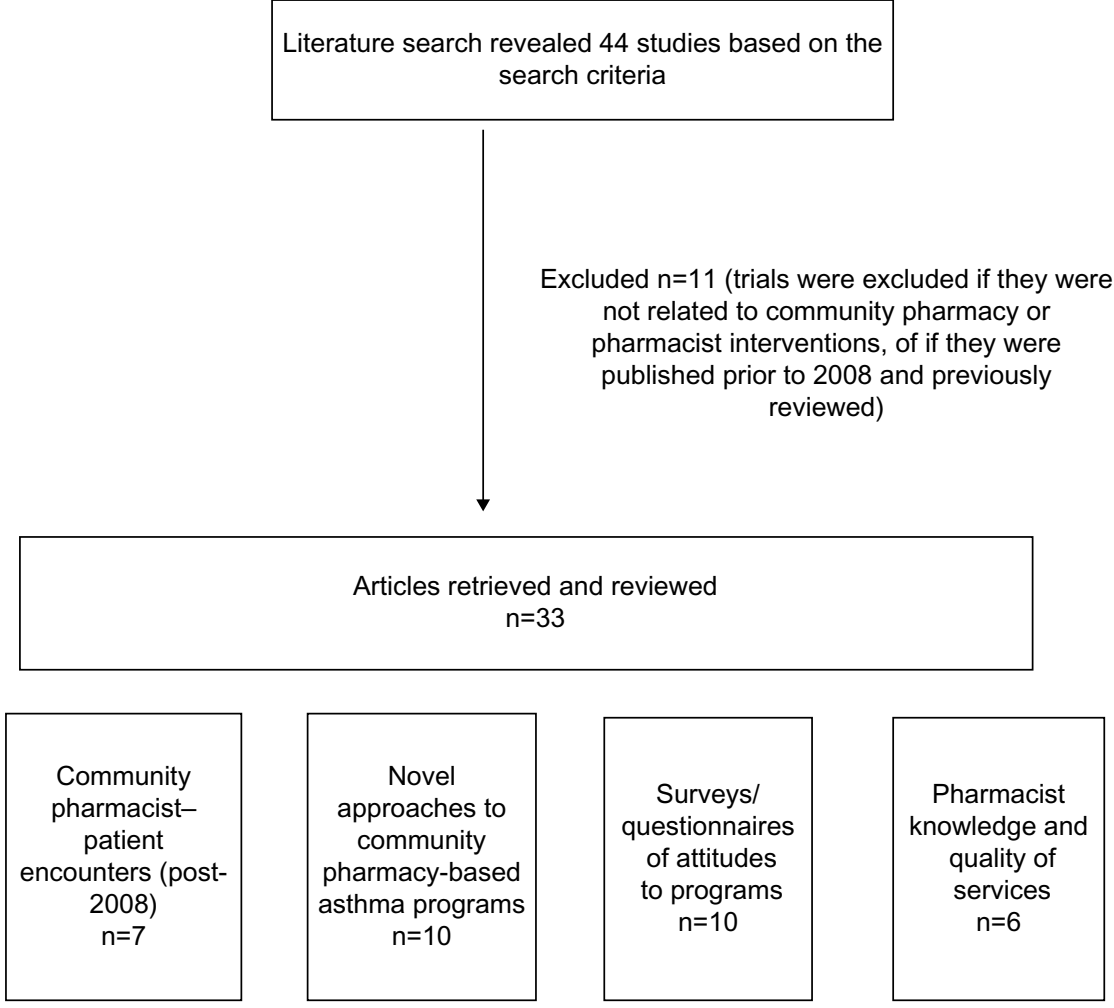

Articles retrieved and reviewed $\mathrm{n}=33$
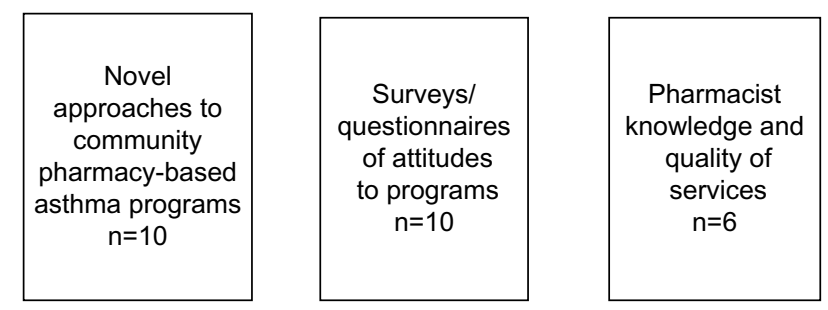

Figure I Study inclusion diagram. Abbreviation: $\mathrm{n}$, number. 
- Novel approaches to community pharmacy-based asthma programs: $n=10$.

- Surveys of pharmacists', physicians', or patients' attitudes: $n=10$.

- Training of pharmacists to provide asthma services: $\mathrm{n}=6$.

The results of each category are summarized below.

\section{Community pharmacist-patient encounters for asthma (post 2008)}

Recent studies have evaluated the impact of community pharmacist interventions on various asthma outcomes such as medication adherence, ${ }^{6-8}$ improving appropriate drug use, ${ }^{9}$ asthma knowledge,${ }^{6,10}$ pulmonary function tests, ${ }^{6}$ and asthma control. ${ }^{7,8,10}$ The results of these studies are summarized in Table 1.

Community pharmacists at one outpatient pharmacy ${ }^{6}$ assessed the effect of providing four structured asthma education visits over 4 months on treatment outcomes. The authors concluded that education by community pharmacists positively impacted subjects' asthma management, knowledge of asthma and device technique, treatment adherence, and attitudes. Strengths of this study included the use of prepared inhalers and Rotahaler ${ }^{\circledR}$ (Cipla Limited, Mumbai, India) to assess the technique of participants. Unfortunately, the authors did not state the number of participating pharmacists that assessed device technique. A fewer number of pharmacists critiquing all patients in the study would decrease possible interrater variability.

A prospective cohort study ${ }^{9}$ evaluated the impact of using a Web-based support tool to identify drug-related problems and to provide a pharmaceutical care intervention versus usual care to patients with asthma or COPD in Dutch community pharmacies. Nineteen potential drug-related problems were defined by current practice guidelines, including overuse of short-acting beta agonist (SABA) medication, suboptimal maintenance therapy with longacting beta agonist (LABA) medications, poor adherence to inhaled corticosteroids (ICS), and inappropriate device use or selection. These problems were translated into algorithms, and a monthly pharmacy report was run using dispensing data to identify eligible adult patients (ages 16-40 years). Pharmacists were encouraged to improve patient device technique, device selection and adherence to maintenance therapies; and any order changes were communicated to patients. Pharmacists in the intervention group (IG) were paid for each patient who entered into the program by the study sponsor (GlaxoSmithKline plc, Brentford, UK) and reimbursed for the cost of the Web-based tool. The primary endpoint was a reduction in oral treatment with high-dose oral corticosteroids or antibiotics. At the IG pharmacies, the prevalence of drug-related problems in all patients (including those not receiving the comprehensive program) decreased by $24 \%-41 \%$ and was significantly less than in the control group (CG) for all problems, except for the cessation of LABA. In the IG patients, 14 of the 19 drug-related problems decreased more than in the CG. Improvements were thought to be due to the initiation of and adherence to maintenance therapy, the cessation of suboptimal medications, and the selection of inhalation devices. The authors concluded that a pharmacist-led intervention improved the appropriateness of drug therapy, and that the benefit was not limited to those patients receiving the comprehensive program.

A strength of this study ${ }^{9}$ is the large sample size and the fact that over 100 pharmacists participated. Limitations include that neither the training of pharmacists to provide the program, nor the protocol for pharmacist-patient encounters was described. Also, pharmacists volunteered to participate in the IG, which could have resulted in some selection bias toward pharmacists with better communication skills or those with more interest in providing pharmaceutical care.

A 6-month trial compared patients in the $\mathrm{CG}$ receiving "usual pharmacist care" $(n=70)$ or a predefined pharmacist intervention (IG; $\mathrm{n}=80) .{ }^{7}$ Patients with well-controlled asthma (Asthma Control Test [ACT] score of 25) or poorly controlled asthma (ACT score $<15$ ) were excluded. Patients in the IG received five scheduled pharmacist visits (at the beginning of the 2-week run-in period, at randomization, and at 1 month, 3 months, and 6 months). Asthma control, pulmonary function, and treatment adherence improved in the IG, but there was no change in severe exacerbations or quality of life (QOL). Limitations of this trial include the fact that activities during the pharmacist visits were not defined, making these interventions difficult to replicate. A study duration of longer than 1 year may have controlled for seasonal variations in asthma control.

An Australian study ${ }^{10}$ enrolled patients in a 6-month intensive asthma service. Patients were recruited by community pharmacists based on the patients' risk of poor asthma control using a brief risk-assessment tool. Patients completed a baseline knowledge questionnaire and attended either three or four visits at their pharmacy. Pharmacists identified each patient's educational needs using a protocolspecific checklist, but the interventions delivered were done at the discretion of the pharmacist. Assistance was available 
Table I Community pharmacist-patient encounters for asthma (post-2008)

\begin{tabular}{ll}
\hline Author (location) & Methods \\
\hline Kumar et al $^{6}$ (India) & Study type: RCT \\
& Duration: 2 months \\
& Number of visits: four \\
& Pharmacist training: not described \\
& $\mathrm{n}$ (patients) $=52$ IG; 54 CG adults \\
& Population: adults with chronic, stable asthma \\
& $\mathrm{n}$ (pharmacies) $=$ I \\
& \\
Ottenbros et al & \\
(the Netherlands) & Study type: prospective cohort \\
& Duration: 9 months \\
& Number of visits: not standardized; based on the \\
& identification of I9 medication-related problems \\
& using a Web-based algorithm \\
& Pharmacist training: not described \\
& $\mathrm{n}$ (patients) $=3,757$ IG; I05,507 CG \\
Population: adults with asthma or COPD & $\mathrm{n}$ (pharmacies) = I07 IG; I05 CG \\
\end{tabular}

Mehuys et $\mathrm{al}^{7}$ (Belgium)

Saini et al ${ }^{10}$ (Australia)

García-Cárdenas et $\mathrm{al}^{8}$ (Spain)
Study type: R, C parallel-group trial

Duration: 6 months

Number of visits: five

Pharmacist training: yes

$\mathrm{n}$ (patients) $=80 \mathrm{IG} ; 70 \mathrm{CG}$

Population: adults with asthma on a maintenance medication (ACT score: 15-24)

$\mathrm{n}$ (pharmacies) $=66$
Outcomes measured

- A higher percentage of correct answers on the Knowledge, Attitudes, and Practices questionnaire in the IG versus $C G$ (no $P$-value provided).

- MDI technique scores significantly improved in the IG versus CG $(P<0.01)$ at visits 2,3 , and 4 .

- Rotahaler ${ }^{\circledR}$ technique scores in the IG significantly improved over scores in the CG $(P<0.01)$.

- Medication adherence scores significantly improved in the IG versus the CG $(P<0.01)$.

- FEV, improved $200 \mathrm{~mL}$ in IG versus CG (no $P$-value).

- Prevalence of drug-related problems decreased by $24 \%-41 \%$.

- Mean number of high-dose oral corticosteroid or antibiotic prescriptions decreased in the IG by an additional average of 0.54 per patient when compared to the CG (no $P$-value available).

- The reduction in high-dose prescriptions (0.92 per patient; 95\% Cl: 0.58-1.25) was mainly seen in those without concomitant maintenance ICS therapy per post hoc analysis.

- ICS prescriptions were started in 10\% of patients with a decreasing number of high-dose corticosteroid or antibiotic prescriptions.

- ACT score increased in the IG versus the CG (mean difference in ACT scores of 2.0) (95\% Cl: 0.I-3.9; $P=0.038)$.

- Symptoms and pulmonary function per asthma diary: - Less nighttime awakenings in the IG versus the CG $(P=0.044)$;

- Less need for rescue medication in the IG versus the $\mathrm{CG}(P=0.012)$;

- Peak expiratory flow rate: NS.

- Number of correct inhalation technique steps was higher in the IG $(P=0.004)$.

- Mean adherence rates (claims data) was higher in the IG $(90.3 \%)$ versus the CG $(74.6 \% ; P=0.016)$, but not different by self-report.

- Number of severe exacerbations: NS.

- Knowledge of Asthma and Asthma Medicine questionnaire: NS.

- QOL questionnaire scores: NS.

- Consumer Asthma Knowledge Questionnaire scores improved from baseline $(P<0.00 \mathrm{I})$, with no difference between groups receiving 3 or 4 visits $(P=0.30)$.

- Group with 6-month follow-up visit had higher knowledge scores $(P=0.02)$ than follow up by questionnaire. Difference was not sustained at 12-month follow up.

- No significant difference in knowledge scores 12 months after completion of service between those with three visits versus four visits $(P=0.80)$.

- Mean ACQ scores decreased from visit I to 2 in both the IG (0.32 points; $S D=0.9 I ; P<0.00 I)$ and $C G(0.16$ points; $S D=0.73 ; P<0.017)$.

- Mean ACQ scores decreased from visit 2 to 3 in the IG only $(P<0.001)$.

- The proportion of patients with controlled asthma significantly increased from baseline (28\%), visit 2 (43\%), and visit 3 (final visit) (58\%) in the IG, but it was not significantly changed in the CG. 
Table I (Continued)

\begin{tabular}{|c|c|c|}
\hline Author (location) & Methods & Outcomes measured \\
\hline & & $\begin{array}{l}\text { - More subjects with asthma were controlled in the } \\
\text { IG versus CG ( } 58.1 \% \text { versus } 46.0 \% \text {; } P=0.028) \text {. } \\
\text { - The proportion of those adherent increased in the IG from } \\
38 \% \text { to } 78.5 \% \text { versus } 39.3 \% \text { to } 52 \% \text { in the CG }(P<0.001) \text {. } \\
\text { - The proportion of patients with correct device technique } \\
\text { was significantly higher in the IG versus the CG } \\
\text { ( } 78.5 \% \text { versus } 52.0 \% \text {; } P<0.00 \text { I). }\end{array}$ \\
\hline
\end{tabular}

Abbreviations: RCT, randomized controlled trial; n, number; IG, intervention group; CG, control group; MDI, metered-dose inhaler; FEV , forced expiratory volume in I second; COPD, chronic obstructive pulmonary disease; Cl, confidence interval; ICS, inhaled corticosteroids; R, randomized; C, controlled; ACT, Asthma Control Test; NS, not significant; $\mathrm{QOL}$, quality of life; $\mathrm{ACQ}$, asthma control questionnaire; $\mathrm{SD}$, standard deviation.

to pharmacists by phone from the research team. At the end of the program, the knowledge questionnaire was repeated. Six-month patient follow up was either performed by an inperson visit or by the mailing of a knowledge questionnaire. All patients completing a 6-month follow up (either by visit or questionnaire) were mailed the knowledge questionnaire at 12 months. The most frequently reported interventions by pharmacists were counseling about triggers and the role of maintenance medications. The pharmacist intervention improved the knowledge of patients with asthma. A follow-up visit at 6 months appeared to slow the rate of knowledge decline after the program, but did not appear to have a lasting effect on knowledge retention. Strengths include that pharmacists were able to tailor interventions to meet the specific needs of each patient, which mimics a real-life approach, the use of follow up after the program (in an attempt to improve asthma knowledge retention), and further follow up at 1 year. Shortcomings of the study ${ }^{10}$ include a rather cumbersome design, limiting the ability to determine which specific interventions might have led to improvements in each patient's asthma knowledge, and a lack of clinical endpoints.

The last study ${ }^{8}$ evaluated whether pharmacist-provided interventions improved asthma control. Pharmacists provided protocol-based educational interventions on asthma control, medication adherence, and device technique. All received copies of the Spanish asthma guidelines. A facilitator visited each pharmacy periodically to assist in compliance to the protocol. Pharmacists in the $\mathrm{CG}$ received instructions via phone about the study protocol and provided usual care. Their participation was monitored by two visits during the study. Patients in the IG were verbally instructed regarding asthma control and received demonstrations and written information regarding inhalation devices. Adherence was assessed and reasons for nonadherence were explored using the Beliefs about Medicines Questionnaire and Health Belief Model. The pharmacist and patient agreed on goals for the next visit.
At baseline, those in the IG had a higher number of subjects with uncontrolled asthma $(P=0.005)$, and they were taking more asthma medicines $(P=0.38) .{ }^{8}$ The percentage of patients with controlled asthma in the CG remained stable from baseline to the study's end. At the end, more patients in the IG had controlled asthma than in the CG. Using an intent-to-treat analysis, the adjusted odds ratio (OR) was 1.94 (95\% confidence interval $[\mathrm{CI}]: 1.06-3.05 ; P=0.32)$. The authors concluded that the educational intervention in a 6-month study significantly improved asthma control, adherence, and patient knowledge.

The study's ${ }^{8}$ strengths include utilizing an individualized approach to adherence education. This allowed the pharmacist to tailor interventions based on each patient's beliefs, barriers to adherence, and personal goals. Including written, verbal, and physical demonstrations of device technique is an effective method for improving device technique, which may improve drug delivery. Limitations of the study include its short duration (6 months), making it difficult to infer whether the outcomes would be sustained, or whether they were potentially influenced by seasonal variations in asthma severity.

Results of these recent studies show that community pharmacists can positively impact QOL, device technique, adherence to maintenance medicine, and disease knowledge of adult patients with asthma. Most of these studies were of short duration (6 months or less). As only one of the studies continued to follow patients, it is less clear if the benefit is maintained over time. One study showed that pharmacists using prescription data can help providers and patients, by increasing the use of ICS and decrease the use of oral corticosteroids and antibiotics.

One pharmacoeconomic study was identified. Authors of the Pharmacy Asthma Care Program analyzed the results of their previously published multisite, randomized, controlled, repeat measures study, ${ }^{11}$ which was originally conducted in Australia in 57 community pharmacies (29 IGs; 26 CGs). In the initial trial, patients randomized to the IG received 
educational sessions over 6 months regarding medications, triggers, device technique, adherence, and to resolve medication-related problems. Close to 400 subjects were recruited $(79 \%$ with severe persistent or uncontrolled asthma). The proportion of patients who were classified as having severe asthma declined from $87.9 \%$ to $52.7 \%$ during the study $(P<0.001)$, while those in the $\mathrm{CG}$ remained unchanged. The proportion of patients in the IG with correct device technique increased significantly $(P<0.001)$, as did the proportion of patients with an action plan $(P<0.001)$. Similarly, adherence $(80 \%-120 \%$ of maintenance medicines) improved (OR: 1.89; 95\% CI: 1.08-3.30).

The factors that were considered to estimate the long-term cost effectiveness of providing (versus not providing) the asthma program ${ }^{12}$ included medication costs, urgent care use, general practitioner (GP) visits, the time for the pharmacist to complete the intervention, and fixed costs (for example, regarding spirometers, software, promotional aids, and training materials). Factors not included were staff training or the impact on absenteeism and productivity from the patients' perspective. A net benefit of quality-adjusted life years (QALYs) of 0.131 was found in the IG over the CG. The net cost was $\$ 758$ Australian dollars (AUD). The estimated cost per QALY gained over 5 years was AUD \$4,753 with an annual pharmacist review. The best- and worse-case scenario was plausibly AUD \$1,611 to AUD \$7,189 per QALY. This type of data is important to support the use and reimbursement of pharmacists for asthma interventions. Importantly, the majority of patients in this study had uncontrolled or severe persistent asthma. Less cost savings would be expected in providing such a comprehensive program to those with mild or better controlled asthma.

\section{Novel approaches to community pharmacy-based asthma programs}

Previously mentioned asthma programs consisted of multiple pharmacist-patient encounters requiring dedicated pharmacist time and patient adherence to visits. Both of these factors can make it challenging for the typical community pharmacy to sustain a program and provide it to a significant percentage of patients. Several recent programs have tried different approaches to address these challenges (see Table 2).

In the first study, ${ }^{13}$ community pharmacists in rural Australia gave a two-part asthma outreach program and assessed the program's impact on asthma knowledge and patient requests for asthma information at community pharmacies. In the first part of the program, trained pharmacists provided an educational program to students in three local high schools. The asthma knowledge of these students was assessed before and after the program. These students then became asthma peer leaders to younger students at their schools. The second part of the program targeted the entire community. A panel of experts discussed asthma topics in a public forum. The younger students also provided creative dance and drama performances based on what they had learned from the peer leaders. The authors concluded that it is feasible for rural community pharmacists to be proactively involved in health promotion.

The strengths of this program ${ }^{13}$ are that pharmacists were trained to use an evidence-based, peer-led educational approach. By using a train-the-trainer tactic, the reach of the program in the community can be increased. Also, this approach was not limited to those with asthma, but it provided information to the general public. Limitations include the small sample size and lack of a CG, randomization, and sustained impact.

Attendance can be a barrier to patient participation in community pharmacy asthma programs. A RCT ${ }^{14}$ assessed the feasibility, acceptability, and initial impact of pharmacist telephone encounters with patients with asthma in a rural underserved population. In addition to asthma education, pharmacists were trained in communication techniques based on the Indian Health Service counseling model. Pharmacists participated in role playing, received feedback, and were certified via the National Asthma Educator Certification Board Exam. Pharmacists in the IG were each assigned nine or ten patients to contact. Three pharmacist telephone consultations were provided regarding asthma self-management and medication use over a 3-month period. Participants' barriers to managing their asthma medications were identified and addressed. The pharmacist reviewed patients' electronic health record and contacted their provider to resolve medication-related problems, if clinically necessary. Patients in the CG received usual care. Baseline and 3-month postintervention telephone surveys were conducted to assess asthma control (ACT), patient activation (Patient Activation Measure), and adherence (eight-item Morisky scale). Patients' perceptions of the program were positive. This study indicates that telepharmacy interventions can have a positive impact on patient adherence and asthma control. This may be a useful method to provide asthma services in situations with limited pharmacy access, such as in rural areas or with patients lacking transportation.

The strengths of the study ${ }^{14}$ include the RCT design, use of standardized questionnaires, and using certified asthma educator (AE-C) pharmacists. The asthma educator certification 


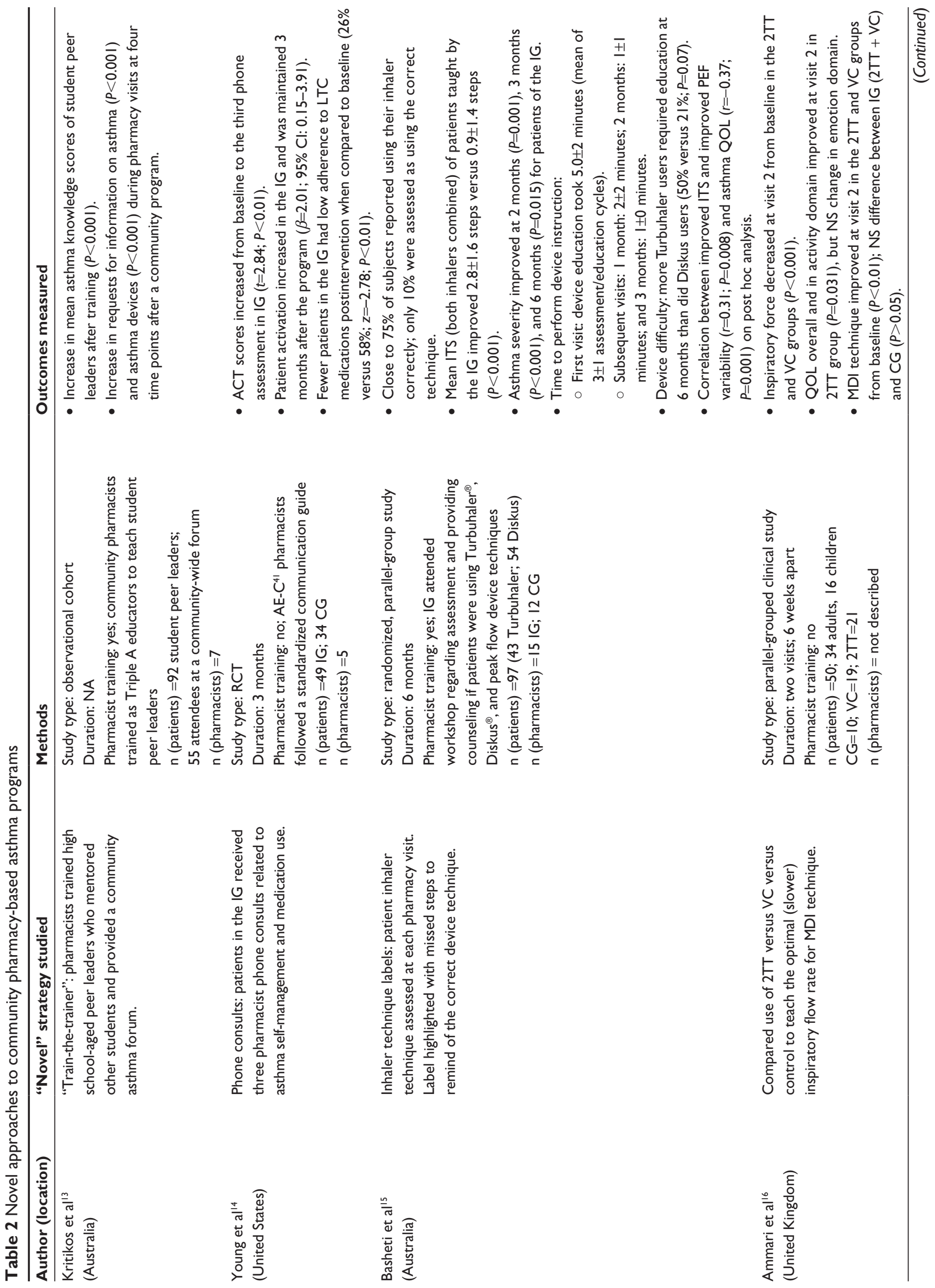




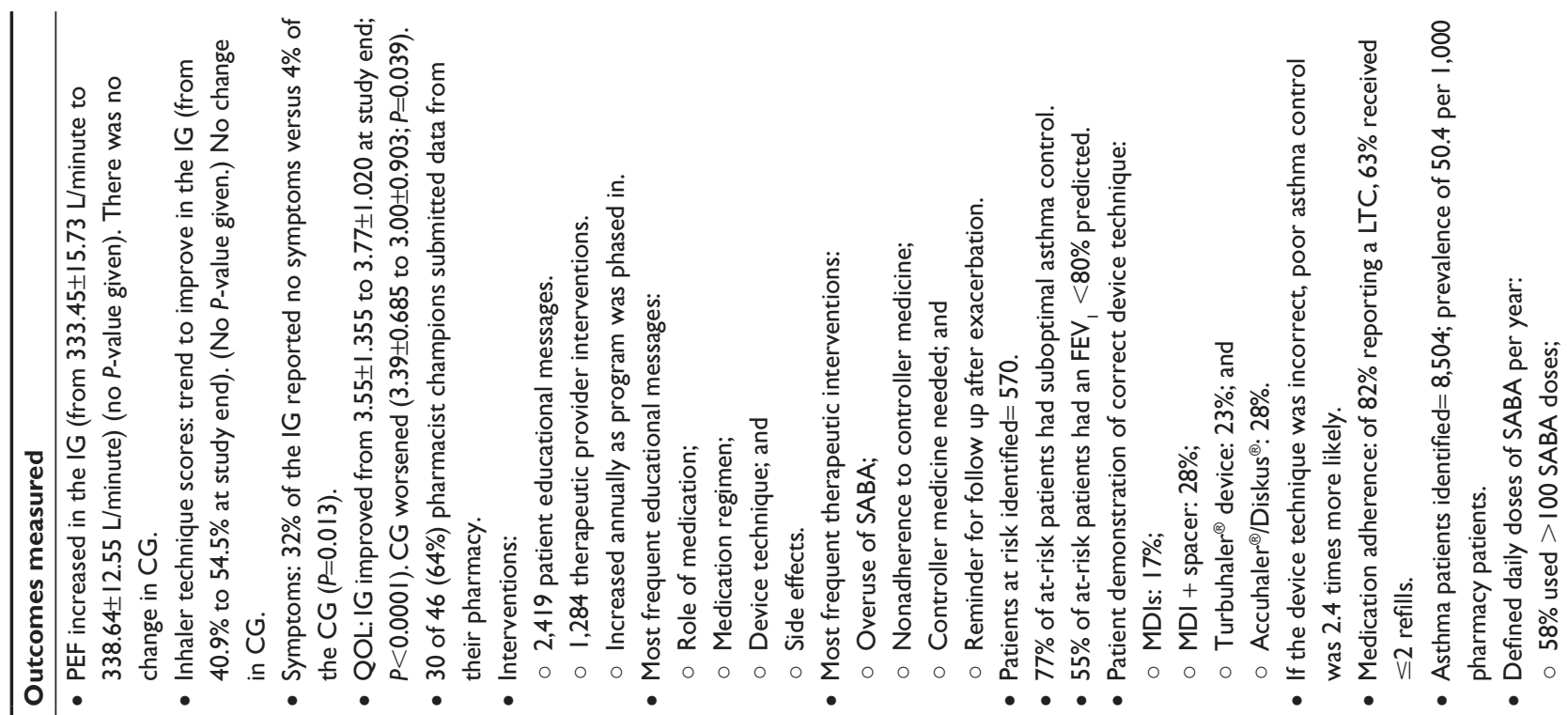

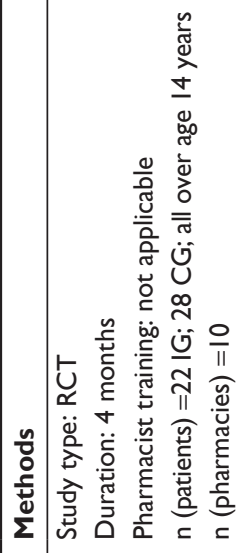

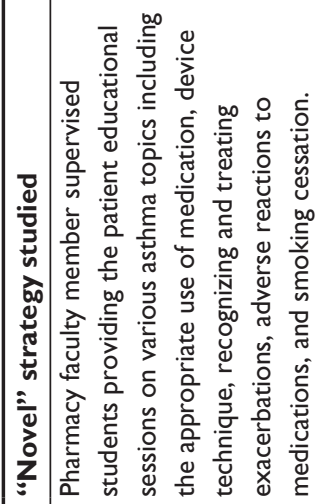

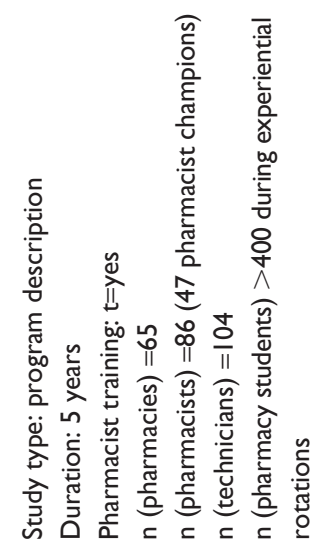

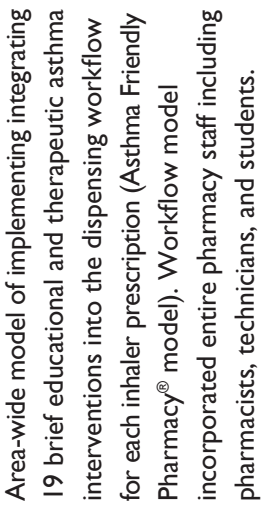

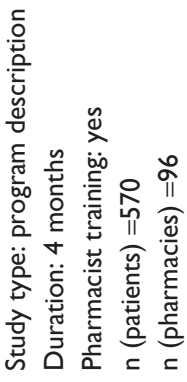
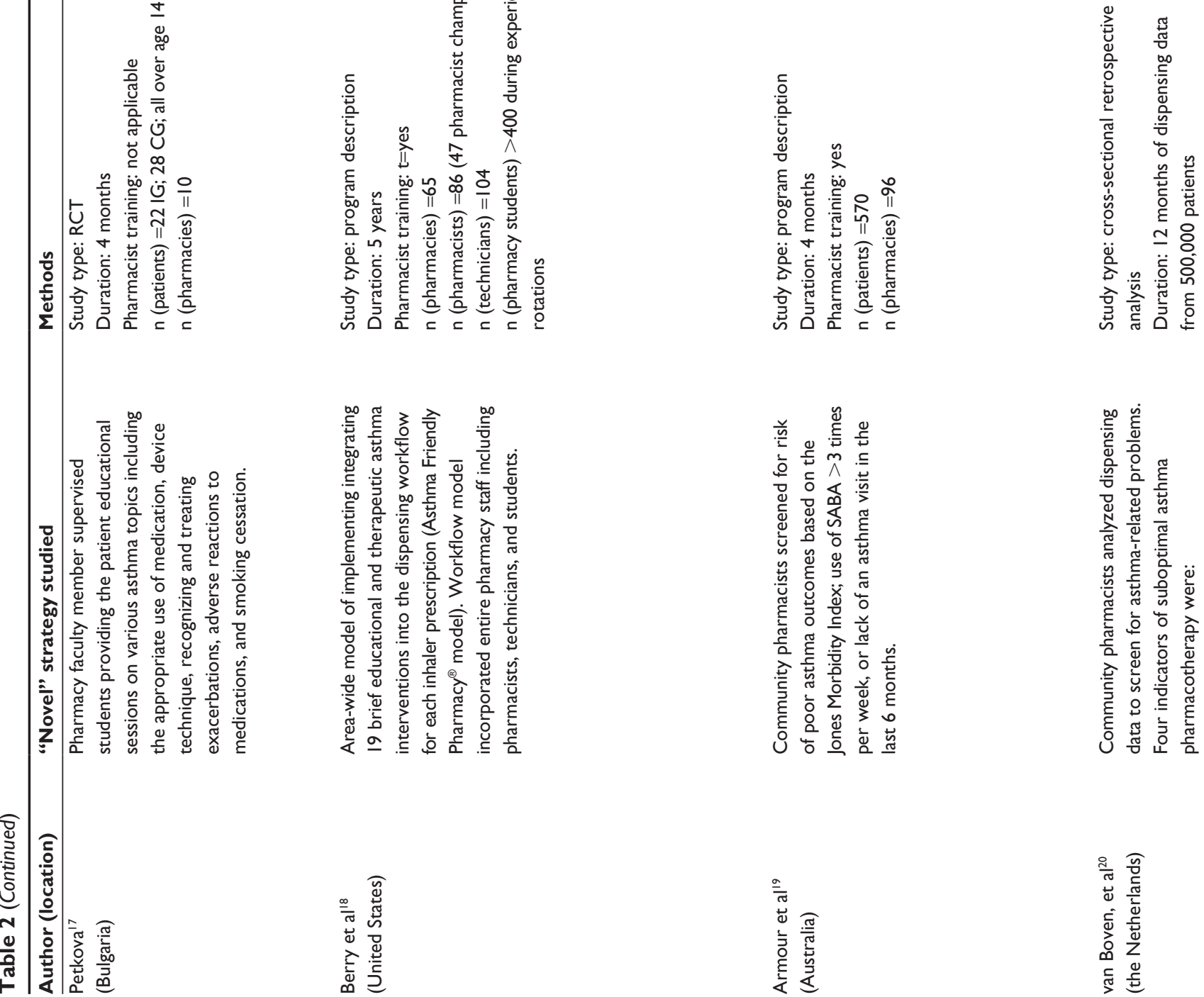

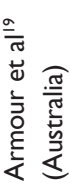



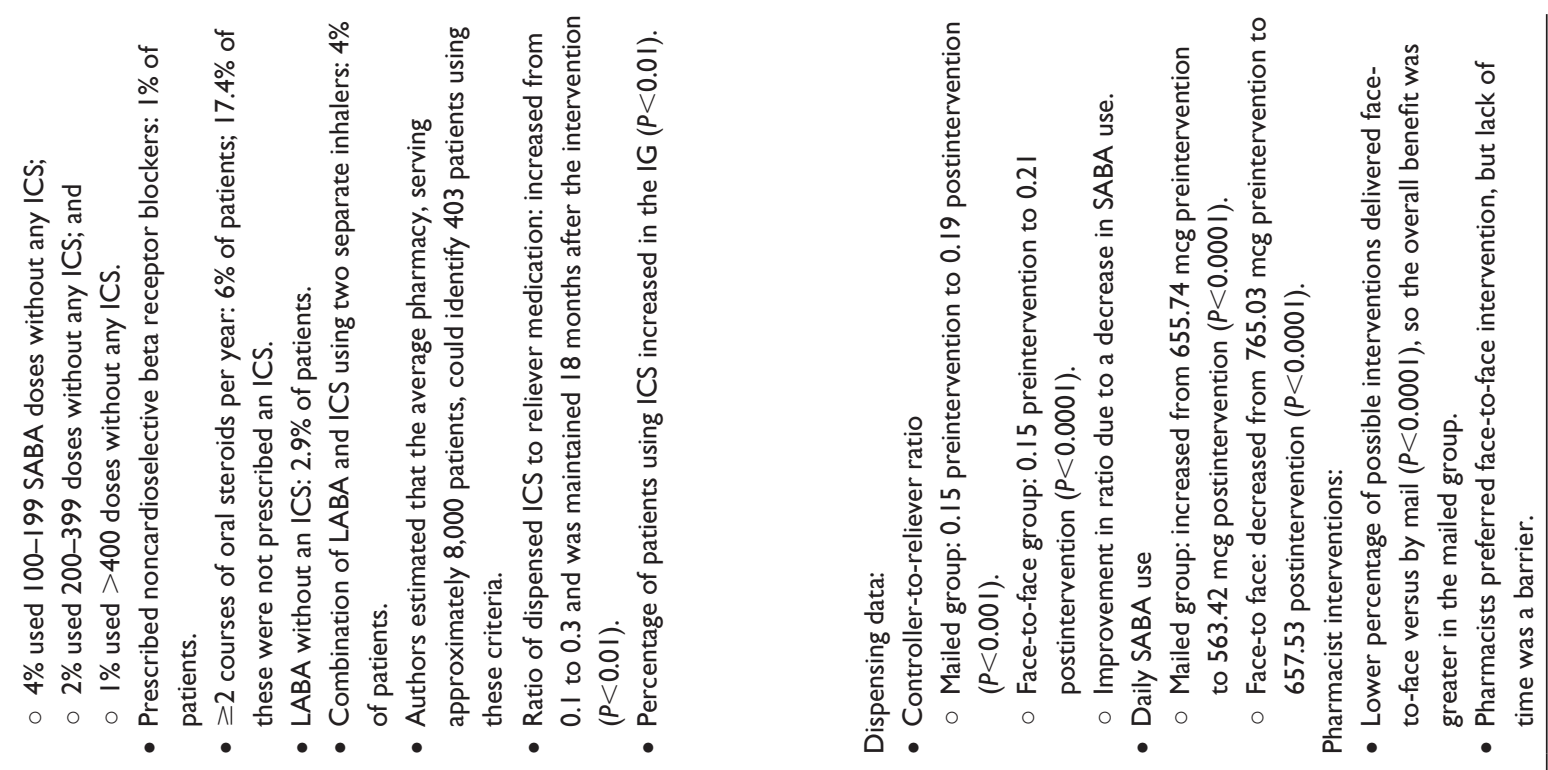

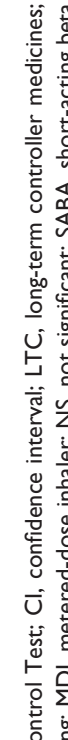

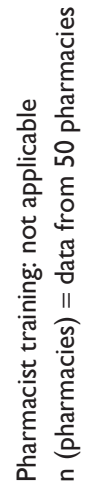
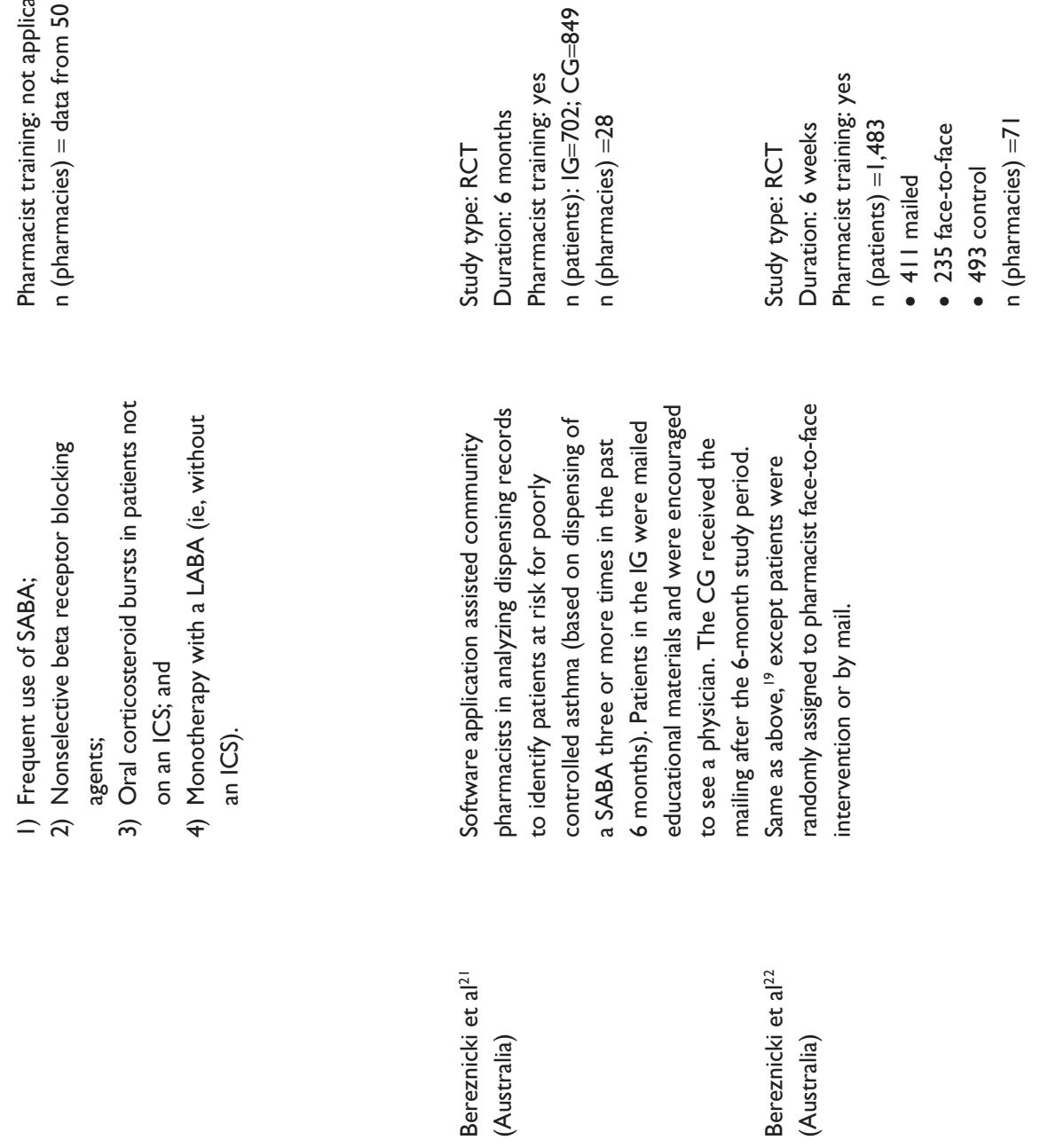
ensures that these pharmacists have passed a nationally recognized and validated examination regarding asthma education. The limitations of this study include the small sample size, short duration, and the lack of an economic analysis.

The amount of time required for a pharmacist to complete a comprehensive program can limit implementation of community pharmacy-based asthma programs. One option is to provide a program focused only on key asthma interventions, such as improving respiratory device technique. Researchers in Australia ${ }^{15}$ evaluated the impact of an educational strategy and a specially designed device label on patient inhaler technique. This study was a single-blinded, cluster, randomized, parallel-group design. Each pharmacist was asked to recruit two to four adult subjects who were receiving either the Turbuhaler ${ }^{\circledR}$ (AstraZeneca plc, London, UK), or Diskus ${ }^{\circledR}$ (GlaxoSmithKline plc) devices for more than 1 month. The technique for subjects in the IG was assessed by the pharmacist and verified by the researchers for half of the subjects. At the first study visit, subjects in the IG received device counseling using up to three cycles of a "Show and Tell" technique. ${ }^{15}$ At the completion of the session, the pharmacist attached a special label to the controller device, highlighting any steps in the technique that were still missed. If the subject was able to complete all steps correctly, the label was attached without highlighting. At each visit, this process of assessing the subjects' device technique and labeling of the dispensed device with missed steps was repeated. For subjects in the $\mathrm{CG}$, the device technique was assessed by researchers at the beginning; it was then reassessed, and the subjects received device counseling by the researcher at study completion. Peak flow variability was the primary outcome.

The study results indicate that by repeating device instructions and using a special reminder label, pharmacists can decrease asthma severity. The amount of time required by the pharmacist to provide repeated device instruction is relatively short. ${ }^{15}$ By utilizing individualized feedback, patients commented that the labels made them feel special and helped them progress towards proper technique. Strengths include the cluster design and teaching both the IG and CG pharmacists how to educate on proper device technique. The use of sticky labels seems to be a low-cost, sustainable way of improving device technique; however, an economic analysis was not reported.

Another clinical study in the United Kingdom ${ }^{16}$ focused on pharmacist instructions in improving respiratory device technique. Subjects with the correct metered-dose inhaler (MDI) technique at baseline comprised the CG. The IG included those with poor MDI technique and was further subdivided into those receiving verbal counseling and those given a 2Tone Trainer tool (2TT) to practice the optimal slow inspiratory flow rate (IFR) lasting at least 5 seconds. Patients in the IG were seen twice; the first visit was to receive verbal training on the correct MDI technique, with an emphasis on achieving the ideal slow IFR. Those in the 2TT group also received additional training (to maintain a single sound on the tool indicating optimal IFR), and they were instructed to practice twice daily with the 2TT just before using their MDI medication. At the second visit 6 weeks later, QOL (by questionnaire), forced expiratory volume in 1 second, and MDI technique were reassessed.

Fifty of 56 subjects completed the study. ${ }^{16}$ The IFR and overall device technique improved in both IGs. QOL improved in the 2TT group. Strengths included enrolling both adults and pediatric patients, and making use of a novel tool to teach proper IFR. Limitations included a small sample size, a short (6-week) follow-up period, and a large percentage of enrolled patients with mild asthma. This may have impacted the results, making a change in QOL scores more difficult to detect. This study provides additional evidence that improving device technique improves asthma-related QOL.

Another way to address the limited time that community pharmacists have to provide patient instruction is to utilize College of Pharmacy faculty and students to deliver interventions. In one study, ${ }^{17}$ four educational sessions regarding device technique and asthma self-management were conducted by a faculty member and ten students over 4 months. Patients in the IG reported more symptom-free days and less use of urgent care services, provider visits, and fewer hospitalizations. Patients in the IG also reported being more satisfied with their pharmacist care. The drawbacks of this study include the small sample size and the short duration of the study (4 months). The impact of this program may have been blunted by primarily enrolling (70\%) subjects with mild asthma.

The Asthma Friendly Pharmacy ${ }^{\circledR}$ Program ${ }^{18}$ took a different approach to reach more patients via asthma messages. Sixteen educational messages based on national asthma guidelines ${ }^{2}$ were integrated into the standard community pharmacy practice. Messages were selected based on the importance and ease of integration into the typical community pharmacy workflow (for example, device technique, adherence to or need for maintenance medications, overuse of SABA, tobacco cessation). The goal was to provide all asthma patients (adults and children) with brief (3-5-minute) messages at the time of medication dispensing. Pharmacists were trained to review a patient's drug use profile and notify physicians by fax of 
medication-related problems. Integration of brief messages into the standard workflow was found to be feasible in a variety of community pharmacy types (chain, independent, and grocery store). This model has the potential to provide messages to a large population of patients with asthma, and it encourages efficient communication of medication-related problems to providers (by fax). However, the authors noted that the sustainability of the program requires significant support from pharmacy managers, assistance so each pharmacy can adapt the general model into their specific workflow, educational materials for pharmacy staff and patients, and a college staff member for ongoing coordination, data collection, and training because of pharmacy staff turnover.

Others have also taken a population approach to improving asthma control in community pharmacies. In Australia, ${ }^{19}$ community pharmacists were trained to identify patients at risk for poor asthma outcomes and factors associated with poor asthma control. Pharmacists classified patients' asthma severity based on asthma symptoms and spirometry, checked maintenance medication adherence via questionnaire and dispensing records, and reviewed device technique. This study enrolled those who self-reported that they had asthma, but it may have included some individuals with COPD. This study demonstrates the potential of trained community pharmacists in effectively performing population-based assessments, so as to identify individuals with potentially poor asthma control for referral to providers. In addition, the authors also noted a connection between poor device technique and decreased asthma control.

Pharmacists are in a unique position to identify medicationrelated problems, such as poor adherence and overuse of quick-relief medications, because of their access to medication dispensing records. Four studies have addressed the potential of the pharmacist to use data mining software in combination with dispensing records to identify patients with asthma who could benefit from a pharmacist asthma intervention.

A group of researchers in the Netherlands ${ }^{20}$ screened 2 years of dispensing data in a large prescription database (over 500,000 patients). The records of 8,504 patients with asthma were identified and screened for medication-related problems. Problems included frequent use of SABA, prescribing of nonselective beta-blocking agents, oral corticosteroid bursts in patients not on an ICS, and monotherapy with a LABA. The authors projected that a typical pharmacy with 8,000 patients and an asthma prevalence of $3.8 \%$ would identify 403 patients with asthma with one of these medication-related problems, and who might potentially benefit from a pharmacist intervention. The results of this study reinforced the potential for pharmacies to utilize their own dispensing data to identify patients who may benefit from pharmacist interventions.

A group of authors in Australia have published two studies that screened dispensing data with a specially designed software program to identify asthma-related medication problems. ${ }^{21,22}$ Patients were flagged as having potentially poorly controlled asthma if the dispensing record indicated three or more SABA prescriptions in a 6-month period. These patients were then eligible for the intervention. ${ }^{21}$ In the first study, ${ }^{21}$ the identified patients were randomized to either the IG $(n=702)$ or the CG $(n=849)$. The results showed an increase in the ratio of dispensed maintenance medication (for example, ICS) to quick-relief medication (SABA) in the IG. There was also a significant increase in the IG patients that were prescribed ICS therapy. The fact that the authors assessed the doses per canister of medication is a strength of this study; however, dispensed SABAs were assumed to be consumed by patients. In addition, misplaced inhalers, sharing of medications with others, and having more than one canister of SABA on hand for both the workplace and home could have skewed the data. Similarly, asthma medications dispensed at another pharmacy were not taken into account.

This same group conducted a similar study ${ }^{22}$ in which eligible patients were randomly assigned to a CG or IG. Pharmacists were paid for attending the educational session and for the postage required for the study protocol. Those in the IG either received a pharmacist-initiated intervention by mail, as in the previous study, ${ }^{21}$ or via face-to-face communication, which allowed for a discussion with the pharmacist. The number of face-to-face interventions completed was significantly less than the number of mailed interventions, which may have offset the overall effectiveness of the face-to-face intervention. Pharmacists reported that they were more confident intervening when provided with data from the dispensing records. Face-to-face patient interaction was preferred by pharmacists, but time was a perceived barrier. The authors concluded that the practice of data mining dispensing records could be implemented more broadly into community pharmacy practice.

These two studies ${ }^{21,22}$ showed that pharmacists can identify a significant population of patients at risk for poor asthma outcomes based on dispensing records. Importantly, these data can potentially be used to improve asthma outcomes by increasing the consistency of therapy with therapeutic guidelines. Providers may not be aware of primary nonadherence to prescribed ICS, or of overuse of SABA 
therapy. The advantages of mailed interventions include the minimal amount of pharmacist time and training required, yet these interventions can still result in significant improvements in the ratio of dispensed maintenance medications to quick-relief medications.

\section{Surveys of pharmacists', physicians', or patients' attitudes regarding community pharmacy services to patients with asthma}

The effectiveness of community pharmacy-based asthma programs requires pharmacist collaboration with and acceptance by providers and patients. Several researchers have explored the perceptions of pharmacists, patients, and physicians toward community pharmacy-based asthma services. These studies are summarized in Table 3.

Bereznicki et al, ${ }^{23}$ in a follow-up report of one of their programs using dispensing data for asthma interventions, ${ }^{21}$ summarized the perceptions of participants: six GPs; ten pharmacists; and ten patients. The perception of pharmacist interventions was generally positive, but patients and GPs felt that the program might be more effective if they better understood the rationale for and benefits of the program, as well as the pharmacists' role and skills in medication management.

Another study ${ }^{24}$ assessed the perceptions of physicians towards pharmacist-provided asthma services. In a prospective study conducted in the US, 16 community and clinic pharmacists sent 60 faxes to prescribers with recommendations regarding their patients' device technique or adherence to maintenance medications. Most (62\%) of the faxed recommendations were to "step up" asthma treatment (for example, add a maintenance medicine) or they offered informational alerts to the provider regarding poor adherence or device technique. Physicians were asked to respond as to whether or not they found the faxed recommendation or information helpful in making clinical decisions. These data indicate that physicians perceived community pharmacist interventions as useful. The strengths of this study ${ }^{24}$ include the use of faxes as a primary means to communicate with prescribers, which allowed both the pharmacists and prescribers to send and view/respond to these recommendations during times that were convenient. Limitations included the low participation rate by pharmacists (of 48 pharmacies, only 33 pharmacists agreed to participate), and the small sample size.

After completing a brief education program, 21 community pharmacists in Canada used a standardized approach to assess 82 patients with asthma $(n=23)$ or COPD $(n=59) .{ }^{25}$ Pharmacists reviewed the role of medications, checked device technique, assessed asthma control/status, identified potential drug-related problems, evaluated medication adherence, and made referrals to health educators. Overall, pharmacists made 59 recommendations. In a satisfaction survey that was administered upon conclusion of the study, providers generally held positive attitudes regarding the pharmacy program, but the number of providers in this study was small $(n=9)$.

The last report found regarding physician perceptions conducted a series of interviews ${ }^{26}$ soliciting GPs' and community pharmacists' perceptions of each other regarding asthma management in the community. Individuals in both professions noted minimal engagement professionally, and they had a limited understanding of each other's role. There were negative relational aspects, and collaboration between the professions was reported as low. Although the sample size in both groups was relatively low, addressing these issues and attitudes could be the focus of future research to improve the effectiveness of pharmacy-based asthma programs.

In one study, ${ }^{27}$ Australian patients were interviewed regarding their preferences for community pharmacistdelivered asthma services. Preferences were compared between patients who had experienced a specialized asthma service at their community pharmacy (experienced patients, $\mathrm{n}=8$ ) and those that had not (naïve patients, $\mathrm{n}=10$ ). The goal was to identify aspects of the services that patients perceived to be important. These data are consistent with the findings obtained by Bereznicki et al, ${ }^{21}$ in that some patients (for example, those who have not received pharmacist services) may have low expectations of their pharmacist. However, patients' expectations of their pharmacist may increase as they experience the benefits of pharmacists' services.

Researchers in Japan ${ }^{28}$ administered a questionnaire to patients who had been counseled by a pharmacist on the use of ICS. Patients were asked about their frequency of SABA use and the degree of asthma control over the last month, as well as about their change in control since starting the ICS. Patients assessed their understanding of the pharmacist's message, how well they communicated with the pharmacist, and their ability to use their inhaler correctly. Based on the patients' assessment, the pharmacists were divided into one of two groups: those with low and high communication ability. Patients reported better outcomes (ie, improvements in asthma) and a higher level of understanding of the information provided when pharmacists were assessed as having a high ability to communicate. This study provides insights 


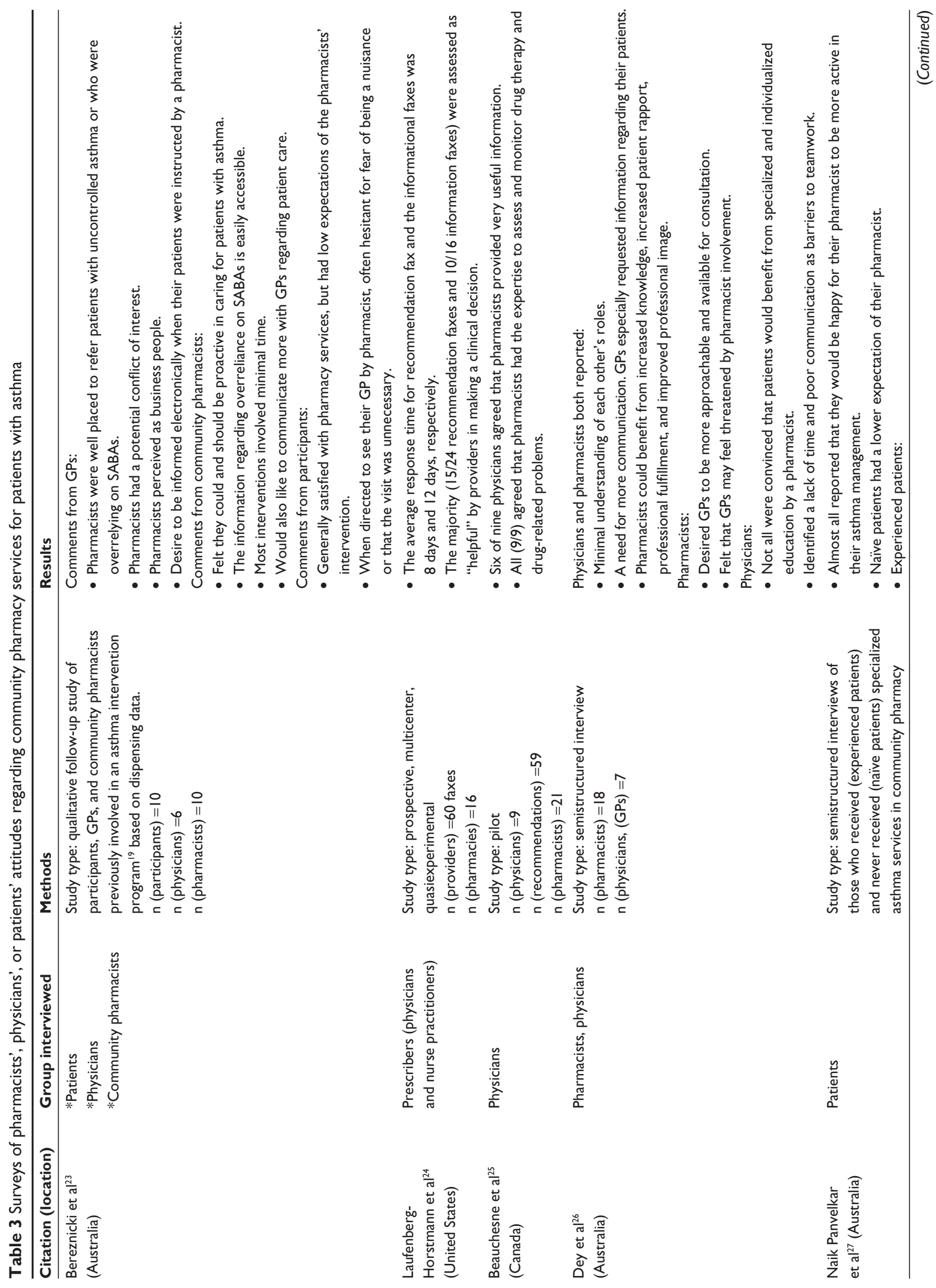




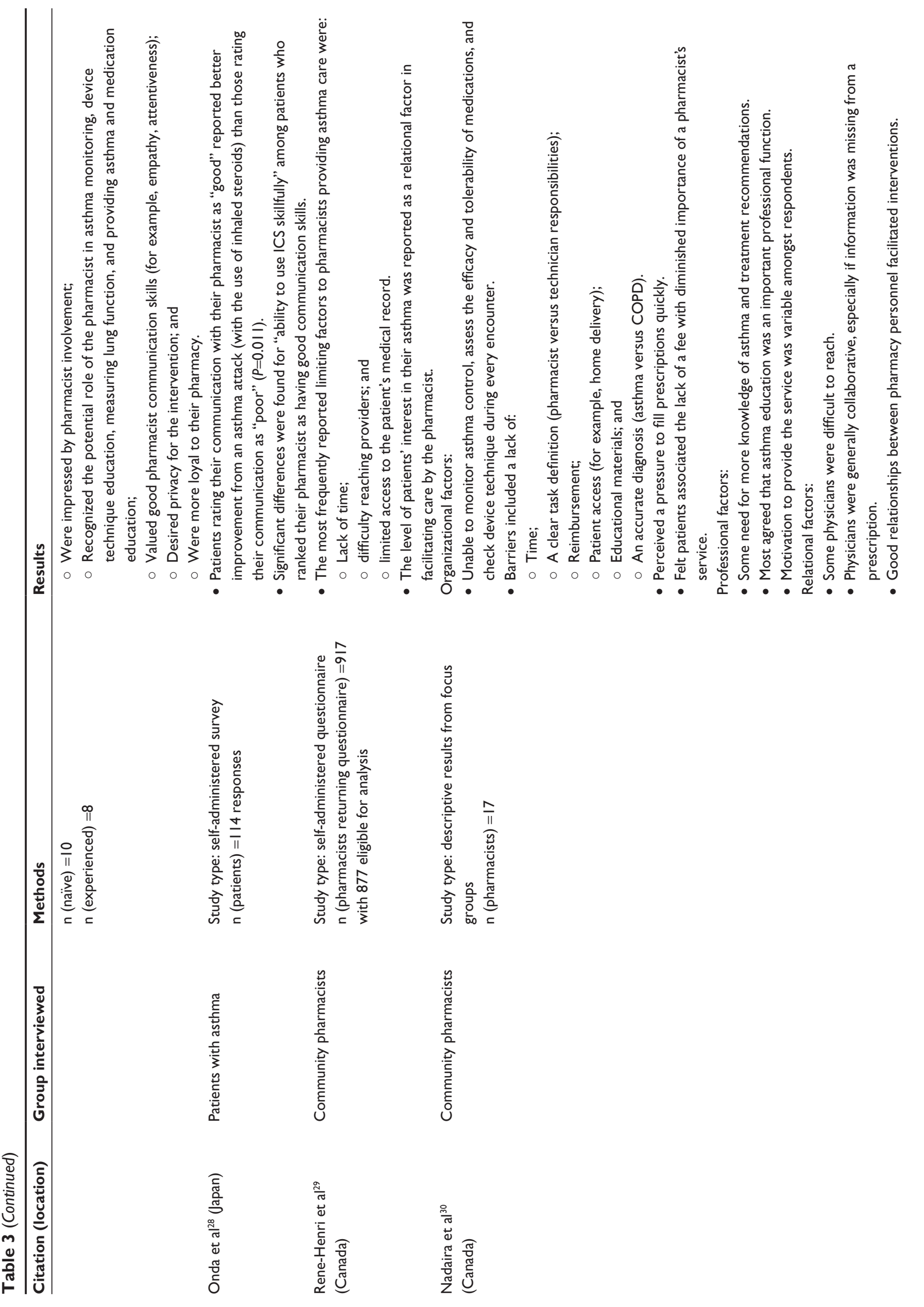



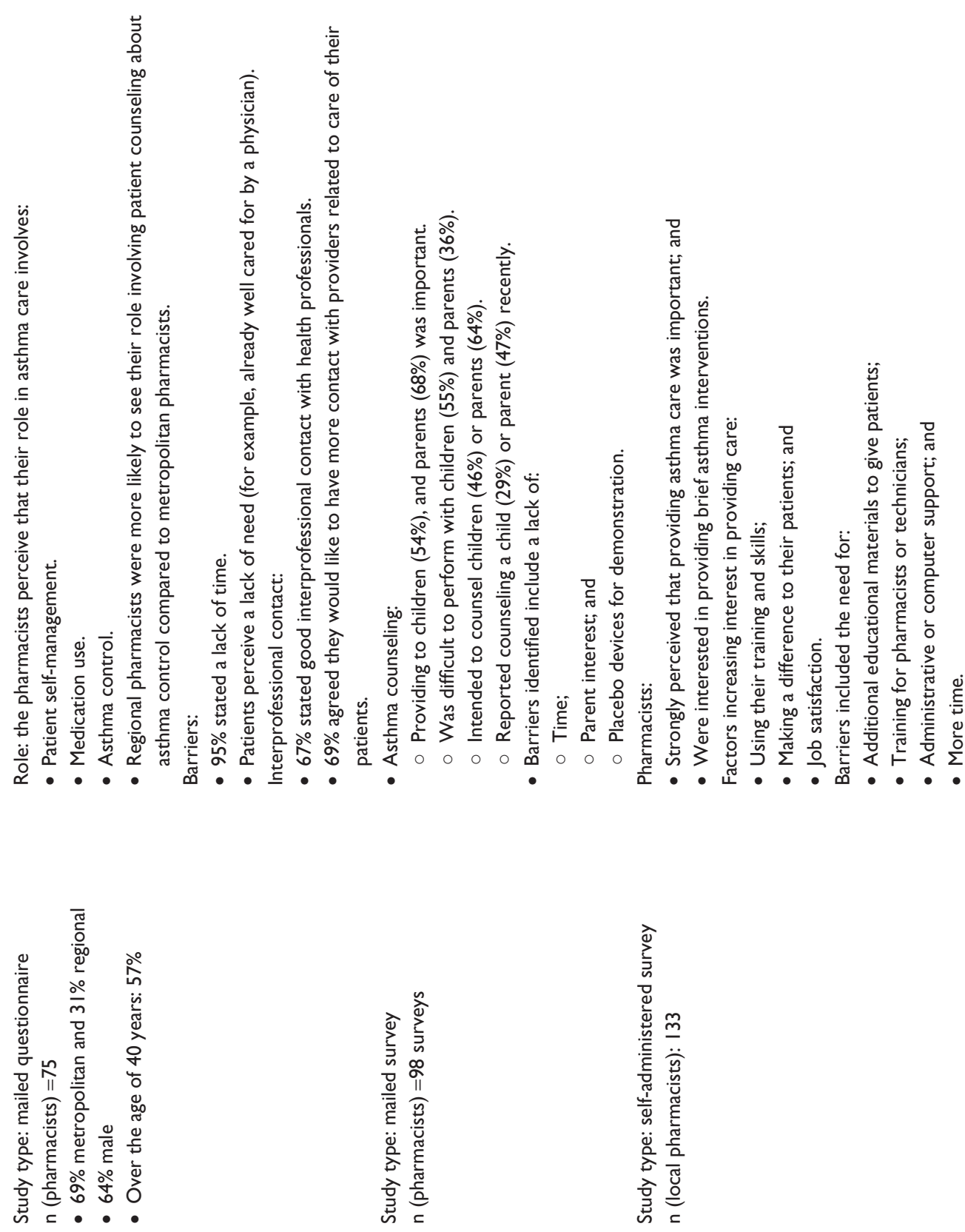

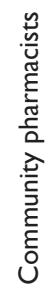

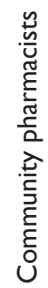

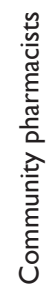
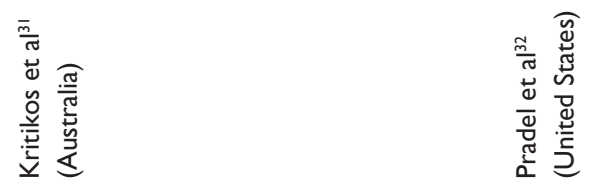

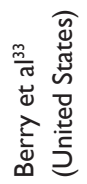

\begin{tabular}{|c|c|c|}
\hline 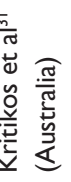 & 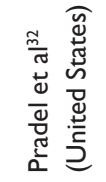 & 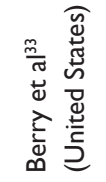 \\
\hline
\end{tabular}


into the importance of communication skills in the overall effectiveness of pharmacist interventions. These data support the development of asthma training programs for pharmacists, which also include communication skills.

More data are available regarding pharmacists' perceptions of their role in providing community-based asthma care. Canadian researchers ${ }^{29}$ surveyed community pharmacists (by mailed questionnaire) to identify organizational, relational, or professional factors influencing their provision of asthma interventions. The two most frequent interventions that were reported were verbal information and device instruction upon first-fill of an asthma medication, the overuse of quick-relief medicine, and nonadherence to maintenance therapy at refills. The authors were able to identify several issues perceived by the pharmacists as limiting the implementation of asthma interventions. Although the data were qualitative, the sample size of pharmacists was fairly large. The authors also proposed possible solutions for these issues to their readers.

In a follow-up to this survey, three focus groups were held at which community pharmacists were asked to identify factors that influenced their provision of asthma care. ${ }^{30}$ These perceptions were grouped into organizational, professional, and relational factors. The focus groups provided a format to further explore pharmacists' perceptions, identify additional barriers, and more explanation for perceptions.

Australian community pharmacists were surveyed regarding the perceptions of their role in asthma care and their relationships to other health professionals. ${ }^{31}$ Of the 84 pharmacists who were mailed the questionnaire, 75 returned it. The barriers to providing care and the interprofessional issues identified were similar to those of other studies. ${ }^{29,30}$ This study also collected information on the pharmacists' perceptions of their role in asthma care. The strengths of the study include the high response rate and the reasonable sample size. The authors did not indicate whether the questionnaire they developed was validated or pretested.

In the US, 389 community pharmacists were surveyed regarding the factors that affected their counseling of children with asthma; 98 responded. ${ }^{32}$ While $81 \%$ of respondents considered demonstrating device technique important, in the previous month, only $25 \%$ actually reported demonstrating device technique to a child and $37 \%$ to a parent. A unique finding of this report is that the intent of the pharmacist to counsel was correlated to the perceived ease of providing counseling, and perceived support for counseling. Also, the intent to counsel was correlated with actually performing patient counseling.
All identified community pharmacists in the St Louis (MO, USA) area were surveyed regarding their perceptions in providing short asthma interventions to their patients. ${ }^{33}$ There was a $79 \%$ response rate representing $91 \%$ of the area pharmacies. Barriers to and perceived perceptions toward providing asthma interventions were similar to those found in the other studies (for example, a lack of time, support, and resources). Strengths of this study included the large response rate for pharmacists in a specific region, and the identification of motivators to providing asthma services, such as being able to make a difference to patients and being able to use their pharmacist training.

Based on these data, future programs that develop collaboration and communication linkages with physicians might strengthen their perceptions of pharmacists' role in providing asthma care. Patients who are recipients of pharmacist-provided care have positive perceptions; those without prior exposure may not perceive it as part of the typical pharmacist role. Pharmacists have a strong interest in providing asthma care, but program models need to be realistic and address the actual and perceived barriers to care, such as a pharmacist's time and skills, while providing adequate support and resources.

\section{Assessment of pharmacist knowledge and quality of asthma services provided}

For clinical services to be effective, providing consistent, high-quality services is important. Studies assessing the quality of asthma services in community pharmacies are summarized in Table 4. Two Australian studies evaluated the quality of the usual pharmacist care for patients with asthma. ${ }^{34,35}$ Community pharmacies were randomized, and a trained simulated patient (SP) visited the pharmacies with a common asthma-related problem. In the first study, ${ }^{34}$ the patient scenario involved the purchase of an over-the-counter SABA inhaler. Although asthma control was assessed in most patients, device technique and frequency of SABA use were less commonly assessed. During $47 \%$ of the visits, the pharmacist on duty was not consulted by the pharmacy staff member that interacted with the patient.

In the second study, ${ }^{35} 155$ community pharmacies were visited by a SP who requested to purchase something for a nonproductive chronic cough. Ideally, the patient should have been assessed as having uncontrolled asthma, and as being in need of a referral to his or her GP. Self-care recommendations regarding asthma by community pharmacy staff in these studies was suboptimal. The results of these studies highlight the need for pharmacists to be proficient in asthma 


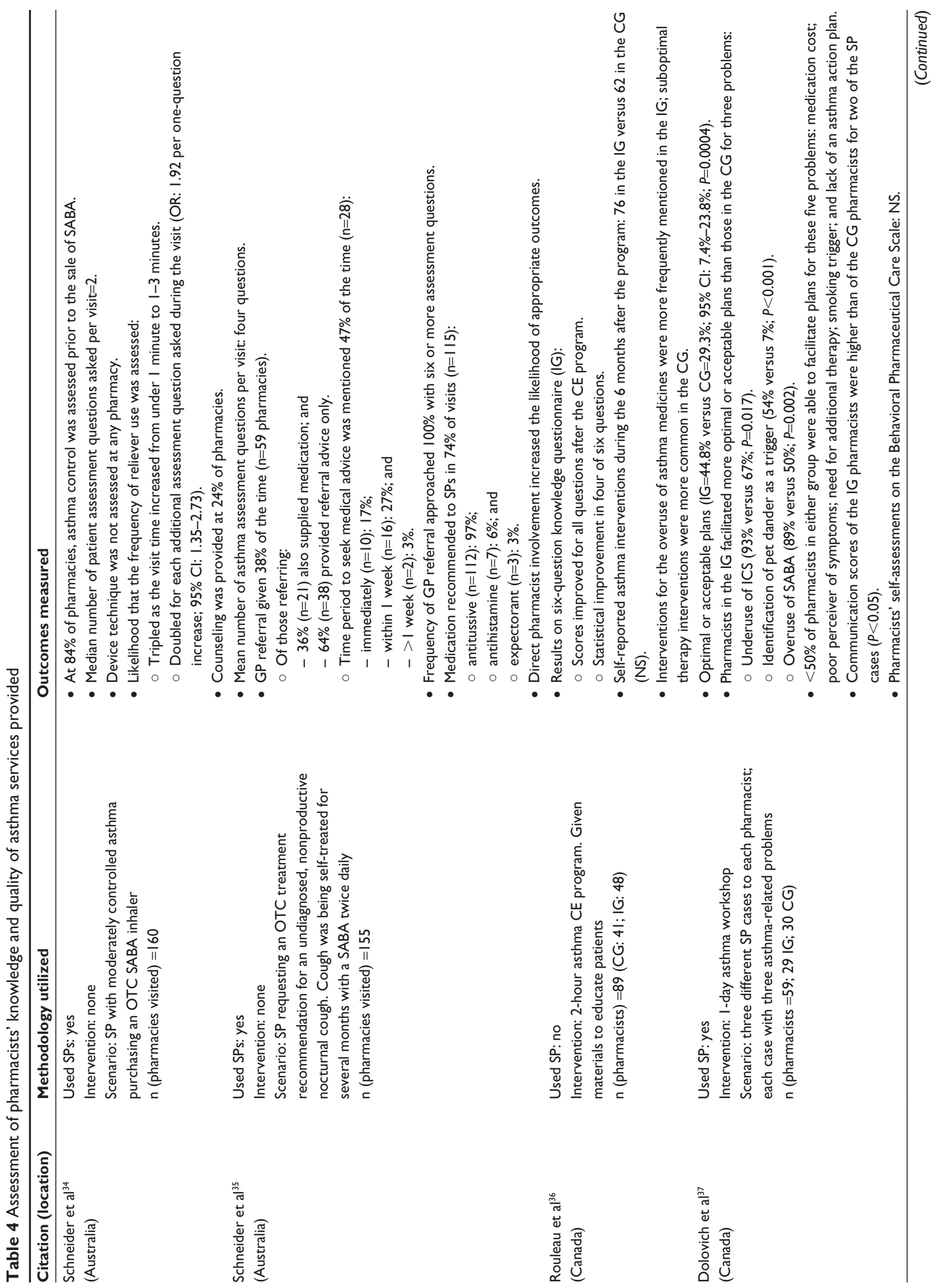


assessment and to develop appropriate recommendations for care. The methodology of assessing the quality of care by a trained SP is a strength; however, the results are dependent on the accuracy of the SP's recording. Some variation was noted in the same case that was acted out by different SPs. The authors also noted the importance of technician training and establishing defined policies and procedures, so that patients have appropriate access to the pharmacist for consultation.

A Canadian pilot study ${ }^{36}$ assessed the impact of a continuing education (CE) program on community pharmacists' knowledge, intervention frequency, and the appropriateness of the asthma medication filled. Pharmacists were enrolled and randomized into either a $\mathrm{CG}(\mathrm{n}=44 ; 41$ completed $)$ or an IG ( $\mathrm{n}=52 ; 48$ completed). Those in the IG attended a CE program prior to the initiation of the study; those in the CG attended it after the study. The CE program involved a lecture and discussion that lasted a total of 2 hours. Guidelines were reviewed and examples were given of situations in which patients should be referred to an asthma education center and receive interventions to improve asthma control. Patient education materials were provided to pharmacists. Both groups completed a baseline assessment of their asthma knowledge before participation in the CE program, and those in the IG repeated the asthma knowledge assessment after the CE program. Both groups reported their asthma intervention data and prescription claims for the 6 months before and after the first group attended the CE program.

Pharmacist knowledge of asthma improved after attending the CE program. However, the number of self-reported asthma interventions was not significantly different between the groups. Only IG pharmacists recommended a referral to an asthma education center. The percentage of prescriptions filled for SABAs, oral corticosteroids, or leukotrienemodifying agents was similar for both groups. Limitations included a small sample size, utilizing a nonvalidated questionnaire, and a short follow-up period. The two different 6-month assessment periods did not control for any seasonal variation in asthma control. These data indicate that a knowledge-based educational program alone may have limited impact on pharmacist behavior. Addressing the barriers and perceptions identified in the pharmacist surveys could increase the number of interventions employed.

A RCT from Canada sought to determine whether an educational program for pharmacists improves their ability to facilitate asthma management or treatment plans. ${ }^{37}$ Prior to the intervention, pharmacists in the IG $(n=29)$ attended a 1-day workshop that included knowledge regarding asthma 
and medications, skills with peak flow monitoring, device technique, patient assessment, developing action plans, and problem solving. A variety of techniques including lectures, role plays, and group work was used. Pharmacists were given a toolkit of key published literature, a tool to prompt them during patient interviews, templates for a letter to communicate with physicians, and a sample patient asthma action plan. Pharmacists in the IG provided $44.8 \%$ appropriate plans compared to $29.3 \%$ in the CG $(95 \% \mathrm{CI}$ : $7.4 \%-23.8 \% ; P=0.0004)$. Strengths included using a randomized, controlled approach. Also, the workshop attended by pharmacists included a variety of teaching techniques that are generalizable to clinical practice, and it addressed some of the pharmacist-identified barriers (for example, it provided patient educational materials, addressed communication skills, device technique, and general asthma knowledge). The use of volunteer pharmacists likely introduced some selection bias, as those highly motivated, more knowledgeable pharmacists might have volunteered for the study. Also, some pharmacists reported that they could detect the SP, which may have heightened their awareness and need for interventions, thus skewing the results.

The use of standardized patients has been utilized in schools of medicine, dentistry, nursing, and pharmacy as a means to improve student learning and to gain practical experience. ${ }^{38}$ A College of Pharmacy in the US used standardized patients to assess a student pharmacist's ability to manage five different medical emergencies. ${ }^{39}$ Students were grouped in threes and assigned roles within a mock community pharmacy. The students generally performed well in the asthma scenario. Using standardized patients can enable student pharmacists to apply their knowledge, as well as practice communication and assessment techniques while being immersed in a stressful situation that may mimic real-world experiences.

In another innovative training program, 157 intern (student or graduate) pharmacists provided an educational program at 136 pharmacies involving 744 pharmacy staff as part of their internship. ${ }^{40} \mathrm{~A}$ health campaign to improve inhaler technique reaching 2,200 patients was also implemented at these pharmacies. The interns had been trained on proper device technique prior to the study. Of the 136 pharmacy sites for the program, 96 IG pharmacies were compared to 97 control pharmacies. SPs visited each pharmacy with a story of worsening asthma control and poor inhaler technique. The appropriate intervention was referral of the SP to their GP for an asthma follow-up visit and the correction of an inhalation technique. The likelihood of referral increased if the patient's asthma control was assessed. Strengths included coordination of a health campaign that was able to reach over 2,000 patients. Weaknesses were again related to SPs and their ability to recall the interaction after it took place.

\section{Discussion}

Pharmacists are uniquely situated within the health care team to care for patients with asthma. In most urban areas, pharmacies and pharmacists are accessible. Patients requiring chronic medications may visit pharmacies as often as every 30-90 days for refills. Brief consultations with a community pharmacist happen without cost or an appointment. As a result, patients with asthma could visit their pharmacist more routinely than their asthma provider. Because they have access to medication use data and patients, pharmacists can identify common medication-related problems such as poor device technique, overuse of quick-relief medications, lack of or nonadherence to maintenance medications, and monotherapy with LABA medications.

Comprehensive asthma programs provided by community pharmacists have improved patients' knowledge of the disease, device technique skills, and QOL. Limited data show that this type of program is cost effective among patients with severe or uncontrolled asthma. The results of the landmark Asheville Project indicated that community pharmacistdriven medication therapy management services increased spending on asthma medications, but they resulted in a net savings of USD \$725/patient/year in direct costs and USD \$1,230/patient/year in indirect costs because of fewer emergency department visits and hospitalizations, and due to lower absenteeism and productivity. ${ }^{4}$ Ideally, if additional data on the impact of such programs on hospitalizations and urgent care visits were available, this would further support the provision of such programs. It is unknown if sufficient benefit would be seen in patients with milder or better controlled asthma to justify the cost of pharmacists' time to provide a comprehensive program. Optimal structure for comprehensive programs, including the number, duration, and intervals of visits, and the content and source of educational materials, and so on, are unknown. It would be helpful if more details regarding the educational protocol are included in future reports to assess the importance of these factors, as well as to facilitate reproducibility of the program by others.

Targeting asthma education to important messages (for example, adherence to maintenance medicines) and skills (for example, device technique) has been correlated with 
improving patient outcomes (for example, QOL); it is also a promising population-based approach. Analysis of medication dispensing data appears feasible and effective in identifying high-risk drug use patterns (for example, nonadherence to maintenance medicines or overuse of SABA). Telephone or mailed informational interventions have also been shown to be effective. Most of these studies have been short term (6 months or less). Studies of longer duration should be performed to assess the impact on seasonal asthma severity. The duration of benefit from asthma interventions is not well studied. However, it appears that repeated interventions are necessary to maintain patients' correct device technique and knowledge over time. ${ }^{2}$ The pharmacoeconomic impact of serial pharmacist-patient visits has only been assessed in one study of patients with uncontrolled asthma. Additional pharmacoeconomic data are needed to confirm this. An assessment of the cost benefit of more targeted approaches should be performed to determine if compensation for such services is justifiable. More data regarding the amount of pharmacist time required to provide interventions would be helpful to assess the full cost and feasibility of program delivery. The quality and consistency of services provided should also be measured.

While pharmacists have been trained in pharmacotherapy and perceive asthma interventions to be important, they perceive several barriers to integrating and providing asthma interventions. It is interesting that the perceptions of pharmacists from different countries are similar. Adequate staffing to provide pharmacist time and supplies (patient education materials, placebo devices, and so on) are necessary to support asthma interventions. Some pharmacists may need refreshers to update their knowledge. Online training programs could provide content, but more than knowledge of asthma and medications is necessary. Skills such as device technique, patient assessment, documentation of patient assessments, and problem solving are required. Patients in one survey ${ }^{28}$ appeared to significantly value pharmacist communication skills. Only one study reported soliciting and addressing patient goals as a part of counseling. ${ }^{8}$ Instruction on communication should include critical skills such as health literacy, individualized goal setting, and motivational interviewing. Pharmacist training to provide asthma services was not detailed in many studies. In addition to didactic lectures, hands-on workshops for device technique, patient simulations, and role playing of common asthma scenarios are methods that pharmacists can use to practice their skills, receive feedback, document competency, and develop confidence. The optimal format and duration of such training courses to develop adequate pharmacist skills and to efficiently deliver a program on a scale that reaches sufficient numbers of pharmacists should be studied. Asthma training should also be incorporated into pharmacy curricula so new pharmacists graduate with such skills. Only one report ${ }^{14}$ mentioned the incorporation of pharmacists that were AE-C. ${ }^{41}$ This multidisciplinary credential could increase the credibility of pharmacist-provided asthma education and encourage the development of pharmacist skills in the area of asthma.

More studies are needed to develop optimal models to integrate asthma interventions into the dispensing workflow. Such integration would assist in ensuring consistency in and sustainability of providing asthma services. Patients and physicians exposed to pharmacist-provided asthma services perceive them to be beneficial. However, a critical mass of pharmacists providing such services routinely to all asthma patients is likely necessary for patients and providers to expect these services from all pharmacists.

\section{Conclusion}

Community pharmacists have been shown to impact patients with asthma. Improvements in knowledge scores, device technique, QOL, asthma control, as well as symptom scores and pulmonary function have been documented after pharmacist interventions with patients. A movement towards "institutionalizing" the provision of routine asthma interventions or encounters is necessary if consistent services are to be given to all patients. Providing consistent quality services would expand both patient and provider perceptions and expectations from pharmacists. Similarly, important interventions related to patient outcomes, such as optimal device technique and adherence to maintenance medications, should be integrated into community pharmacy workflow. Furthermore, a thorough cost analysis could assist with reimbursement models for third-party payers, which may foster the acceptance of improved patient education and interventions in the pharmacists' daily workflow. For such undertakings to become realized, a critical mass of pharmacists educating, interacting with, and documenting outcomes in their patients with asthma in the community is vital.

\section{Disclosure}

The authors report no conflicts of interest in this work.

\section{References}

1. Centers for Disease Control and Prevention [webpage on the Internet]. Survey reveals growing national impact of asthma. Atlanta, GA: Centers for Disease Control and Prevention; 2012. Available from: http://www. cdc.gov/media/releases/2012/p0515_asthma_impact.html. Accessed March 10, 2014. 
2. National Heart, Lung, and Blood Institute Health Information Center. National Asthma Education and Prevention Program. Expert Panel Report 3: Guidelines for the Diagnosis and Management of Asthma. Bethesda, MD: National Heart, Lung, and Blood Institute Health Information Center; 2007. Available from: http://www.nhlbi.nih.gov/ guidelines/asthma/asthgdln.pdf. Accessed March 10, 2014.

3. Healthy People 2020 [webpage on the Internet]. Respiratory Diseases: Asthma. Washington, DC: US Department of Health and Human Services; 2014. Available from: http://www.healthypeople.gov/2020/ topicsobjectives2020/objectiveslist.aspx?topicId=36. Accessed March 12, 2014.

4. Bunting BA, Cranor CW. The Asheville Project: long-term clinical, humanistic, and economic outcomes of a community-based medication therapy management program for asthma. J Am Pharm Assoc (2003). 2006;46(2):133-147.

5. Benavides S, Rodriguez JC, Maniscalco-Feichtl M. Pharmacist involvement in improving asthma outcomes in various healthcare settings: 1997 to present. Ann Pharmacother. 2009;43(1):85-97.

6. Kumar A, Adepu R, Parthasarathi G, Mahesh PA. Impact of community pharmacist provided patient education in asthma patients on treatment outcomes. Indian Journal of Pharmaceutical Education and Research. 2009;43(2):125-133.

7. Mehuys E, Van Bortel L, De Bolle L, et al. Effectiveness of pharmacist intervention for asthma control improvement. Eur Respir J. 2008;31(4):790-799.

8. García-Cárdenas V, Sabater-Hernández D, Kenny P, Martínez-Martínez F, Faus MJ, Benrimoj SI. Effect of a pharmacist intervention on asthma control. A cluster randomised trial. Respir Med. 2013;107(9): 1346-1355.

9. Ottenbros S, Teichert M, de Groot R, et al. Pharmacist-led intervention study to improve drug therapy in asthma and COPD patients. Int J Clin Pharm. 2014;36(2):336-344.

10. Saini B, LeMay K, Emmerton L, et al. Asthma disease managementAustralian pharmacists' interventions improve patients' asthma knowledge and this is sustained. Patient Educ Couns. 2011;83(3): 295-302.

11. Armour C, Bosnic-Anticevich S, Brillant M, et al. Pharmacy Asthma Care Program (PACP) improves outcomes for patients in the community. Thorax. 2007;62(6):496-502.

12. Gordois A, Armour C, Brillant M, et al. Cost-effectiveness analysis of a pharmacy asthma care program in Australia. Disease Management and Health Outcomes. 2007;15(6):387-396.

13. Kritikos V, Saini B, Bosnic-Anticevich SZ, et al. Innovative asthma health promotion by rural community pharmacists: a feasibility study. Health Promot J Austr. 2005;16(1):69-73.

14. Young HN, Havican SN, Griesbach S, Thorpe JM, Chewning BA, Sorkness CA. Patient and pharmacist telephonic encounters (PARTE) in an underserved rural patient population with asthma: results of a pilot study. Telemed J E Health. 2012;18(6):427-433.

15. Basheti IA, Armour CL, Bosnic-Anticevich SZ, Reddel HK. Evaluation of a novel educational strategy, including inhaler-based reminder labels, to improve asthma inhaler technique. Patient Educ Couns. 2008;72(1):26-33

16. Ammari WG, Chrystyn H. Optimizing the inhalation flow and technique through metered dose inhalers of asthmatic adults and children attending a community pharmacy. J Asthma. 2013;50(5):505-513.

17. Petkova VB. Pharmaceutical care for asthma patients: a community pharmacy-based pilot project. Allergy Asthma Proc. 2008;29(1) $55-61$.

18. Berry TM, Prosser TR, Wilson K, Castro M. Asthma friendly pharmacies: a model to improve communication and collaboration among pharmacists, patients, and healthcare providers. J Urban Health. 2011;88 Suppl 1:113-125.

19. Armour CL, Lemay K, Saini B, et al. Using the community pharmacy to identify patients at risk of poor asthma control and factors which contribute to this poor control. J Asthma. 2011;48(9): 914-922.
20. van Boven JF, Hiddink EG, Stuurman-Bieze AG, Schuiling-Veninga CC, Postma MJ, Vegter S. The pharmacists' potential to provide targets for interventions to optimize pharmacotherapy in patients with asthma. Int J Clin Pharm. 2013;35(6):1075-1082.

21. Bereznicki B, Peterson G, Jackson S, Walters EH, Gee P. The sustainability of a community pharmacy intervention to improve the quality use of asthma medication. J Clin Pharm Ther. 2011;36(2): 144-151.

22. Bereznicki BJ, Peterson G, Jackson S, et al. Uptake and effectiveness of a community pharmacy intervention programme to improve asthma management. J Clin Pharm Ther. 2013;38(3):212-218.

23. Bereznicki B, Peterson G, Jackson S, et al. Perceived feasibility of a community pharmacy-based asthma intervention: a qualitative follow-up study. J Clin Pharm Ther. 2011;36(3):348-355.

24. Laufenberg-Horstmann E, DeVore E, Bassuener K. The Coulee Region Community Pharmacy Asthma Intervention study. J Am Pharm Assoc (2003). 2006;46(6):738-746; quiz 746-747.

25. Beauchesne MF, Bercier D, Julien-Baker F, Lalonde L, Boileau R, Blais L. Community pharmacy-based medication assessment program for asthma and chronic obstructive pulmonary disease. Can J Pharm $J$ (Ott). 2012;145(2):70-71.

26. Dey RM, de Vries MJ, Bosnic-Anticevich S. Collaboration in chronic care: unpacking the relationship of pharmacists and general medical practitioners in primary care. Int J Pharm Pract. 2011;19(1): 21-29.

27. Naik Panvelkar P, Armour C, Saini B. Community pharmacy-based asthma services - what do patients prefere? J Asthma. 2010;47(10): 1085-1093.

28. Onda M, Sakurai H, Hayase Y, Sakamaki H, Arakawa Y, Yasukawa F. Effects of patient-pharmacist communication in the treatment of asthma. Yakugaku Zasshi. 2009;129(4):427-433.

29. René-Henri N, Khamla Y, Nadaira N, et al. Community pharmacists' interventions in asthma care: a descriptive study. Ann Pharmacother. 2009;43(1):104-111.

30. Nadaira N, Ouellet C, René-Henri N, et al. Factors influencing a community pharmacist's interventions in asthma care. Canadian Pharmacists Journal. 2009;142(5):240-246.

31. Kritikos VS, Reddel HK, Bosnic-Anticevich SZ. Pharmacists' perceptions of their role in asthma management and barriers to the provision of asthma services. Int J Pharm Pract. 2010;18(4):209-216.

32. Pradel FG, Obeidat NA, Tsoukleris MG. Factors affecting pharmacists' pediatric asthma counseling. J Am Pharm Assoc (2003). 2007;47(6):737-746.

33. Berry TM, Ellis MA, Durrett AJ, Prosser TR. Community pharmacists' attitudes toward providing asthma counseling to ambulatory patients. International Pharmaceutical Abstracts. 2003;38: P-528-E.

34. Schneider CR, Everett AW, Geelhoed E, Kendall PA, Clifford RM. Measuring the assessment and counseling provided with the supply of nonprescription asthma reliever medication: a simulated patient study. Ann Pharmacother. 2009;43(9):1512-1518.

35. Schneider CR, Everett AW, Geelhoed E, et al. Provision of primary care to patients with chronic cough in the community pharmacy setting. Ann Pharmacother. 2011;45(3):402-408.

36. Rouleau R, Beauchesne MF, Laurier C. Impact of a continuing education program on community pharmacists' interventions and asthma medication use: a pilot study. Ann Pharmacother. 2007;41(4): 574-580.

37. Dolovich L, Sabharwal M, Agro K, et al. The effect of pharmacist education on asthma treatment plans for simulated patients. Pharm World Sci. 2007;29(3):228-239.

38. Sales I, Jonkman L, Connor S, Hall D. A comparison of educational interventions to enhance cultural competency in pharmacy students. Am J Pharm Educ. 2013;77(4):76.

39. Robinson JD, Bray BS, Willson MN, Weeks DL. Using human patient simulation to prepare student pharmacists to manage medical emergencies in an ambulatory setting. Am J Pharm Educ. 2011;75(1):3. 
40. Schneider CR, Everett AW, Geelhoed E, et al. Intern pharmacists as change agents to improve the practice of nonprescription medication supply: provision of salbutamol to patients with asthma. Ann Pharmacother. 2010;44(7-8):1319-1326.
41. National Asthma Educator Certification Board (NAECB) [webpage on the Internet]. National Asthma Educator Certification Board. Gilbert, AZ: National Asthma Educator Certification Board; 2013. Available from: http://naecb.com/. Accessed March 12, 2014.

\section{Publish your work in this journal}

Integrated Pharmacy Research and Practice is an international, peer-reviewed, open access, online journal, publishing original research, reports, reviews and commentaries on all areas of academic and professional pharmacy practice. This journal aims to represent the academic output of pharmacists and pharmacy practice with particular focus on integrated care. All papers are carefully peer reviewed

Submit your manuscript here: http://www.dovepress.com/integrated-pharmacy-research-and-practice-journal to ensure the highest standards as well as ensuring that we are informing and stimulating pharmaceutical professionals. The manuscript management system is completely online and includes a very quick and fair peer-review system, which is all easy to use. Visit http://www.dovepress.com/testimonials.php to read real quotes from published authors. 